\title{
The Case of Lower Jaw Resection due to Medication-Induced Osteonecrosis Mronj
}

\author{
Tsolov RB ${ }^{1^{*}}$, Yordanov GY ${ }^{2}$, Dushkova TS $^{3}$ \\ ${ }^{I}$ Clinic of Maxillofacial Surgery, St. George University Hospital, Plovdiv, Bulgaria \\ ${ }^{2}$ Department of Allergology, Physiotherapy and Clinical radiology, Faculty of Dental Medicine, Medical \\ University of Plovdiv, Bulgaria \\ ${ }^{3}$ Private dental practice, General dental practitioner, Plovdiv, Bulgaria
}

*Corresponding Author: Dr. Rosen B. Tsolov, Clinic of Maxillofacial Surgery, St. George University Hospital, Plovdiv, Bulgaria.

\begin{abstract}
Medicinal-related osteonecrosis of the jaw (MRONJ) is a serious side effect consisting of progressive bone destruction in the maxillofacial region. In this report, we present a case of a 73-year-old female patient who, after surgery of breast cancer and long-term antibiotic treatment with osteotropic antibiotics, is admitted for treatment at a hospital where she is diagnosed with mandibular resection following medication-induced osteonecrosis. The patient underwent surgical intervention in which the the extraoral fistula is excised, and hemicarticulation of the lower jaw to the right is performed.
\end{abstract}

Keywords: Denosumab, bisphosphonates, osteonecrosis, MRONJ, surgerical tretement

\section{INTRODUCTION}

Medicinal-related osteonecrosis of the jaw (MRONJ) is a serious side effect consisting of progressive bone destruction in the maxillofacial region.Its linked to the uses of different type of medications, mainy from the group of aminocontaining Bisphopshoantes (such as Zoledronate) [1], and also human monoclonal antibodies (such as Denosumab/Xgeva). Initially, medical oncologists have formed the perception that necrosis is a rare side effect. Ignorance of the effects of these agents, an underestimation of dental remediation, and a high risk of necrosis after dental extraction, have made MRONJ a common pathology [2]. Scientific evidence supports the prevailing view that Zoledronic acid has highest risk-potential [3]. The risk of MRONJ in cancer patients receiving zoledronate is $1 \%$ (100 cases in 10,000 patients) and is 50-100 times higher than the placebo group. With Denosumab, the risk of MRONJ ranges from $0.7 \%$ to $1.9 \%$ (70-90 cases in 10,000 patients) and is comparable to zoledronate [4]. Denosumab has an earlier incidence of necrosis than zoledronate and pamidronate, with an average of 14 doses.
Zoledronate replacement with Denosumab is a risk factor for the development of necrosis. Bisphosphonates and Denosumab additively increase the risk of necrosis [5].

\section{CASe Report}

This case repors presents a 73-year-old woman who underwent surgery for breast cancer 6 years ago, completely removing her left breast. Two years after the intervention of the scan and body scintigraphy, bone metastases were detected. She was prescribed treatment with Xgeva. In early 2018, after extraction of a tooth, osteonecrosis of the mandible was diagnosed. The patient underwent continuous antibiotic treatment with osteotropic antibiotics, including consulting with maxillofacial specialists in New Zealand. At the beginning of 2019 she made a CT scan, which outlines the boundaries of the osteonecrotic process(Fig.1). For one year, the patient complained periodically of inflammatory processes in the field of osteonecrosis, undergoing multiple intraoral incisions. A few months before our intervention, an extraoral fistula was obtained, accompanied by constant pain and a putrid odor. Preoperatively, a CT 
scan was performed on the head, plus a reconstructive image, which revealed that the osteonecrotic lesion reached the incisor of the mandible to the right of the chin. Based on this study and clinical data, a decision was made to perform surgery involving hemiexarticulation plus excision of the extraoral skin fistula.
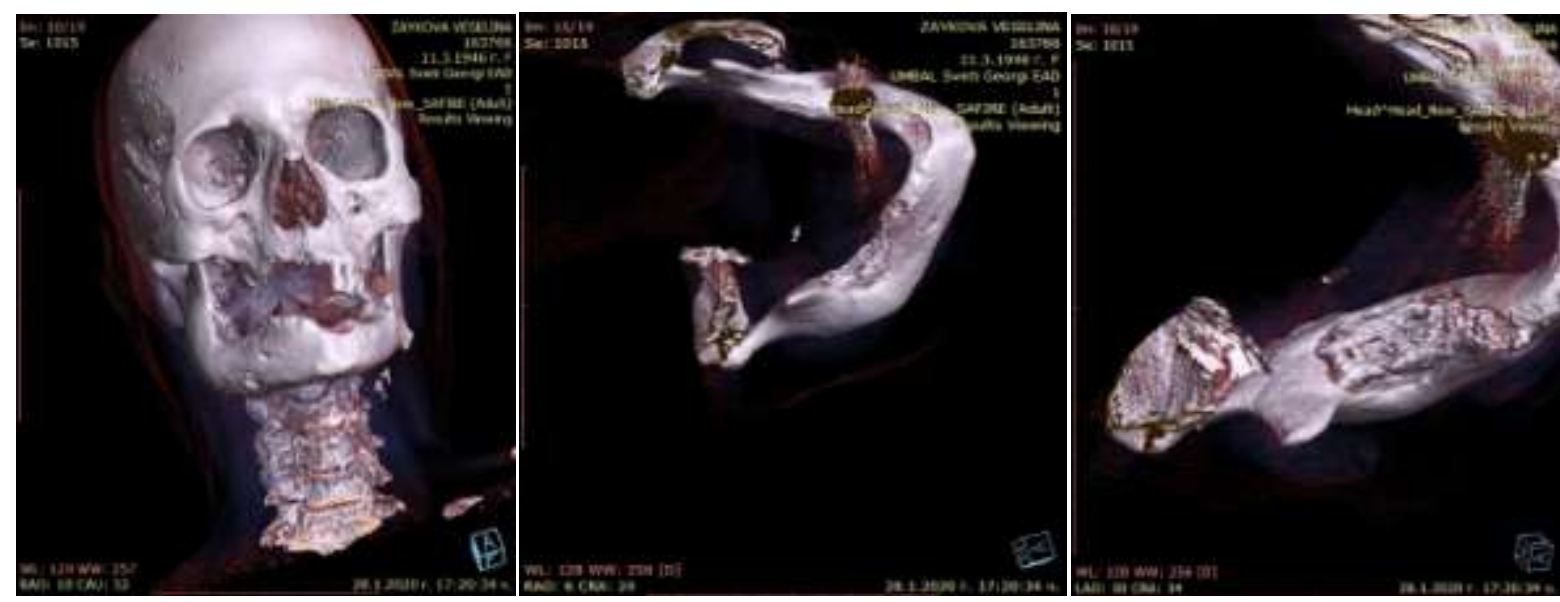

Fig.1. CT Scan before treatment

The blood type - B RF positive was obligatory tested before surgery, two erythrocyte masses were poured preoperatively, and the patient received 12 Tothema ampoules due to low hemoglobin 86. Upon reaching hemoglobin values of 112 , the patient underwent surgery underanesthesia, excision in tight of the extraoral fistula was performe (Fig.2), with consecutivehemicarticulation(Fig.3,4) of the mandible to the right. No cervical dissection of the regional lymphatic basin was necessary because of lack of data on relevant lymph nodes in the cervical lymphatic pool.

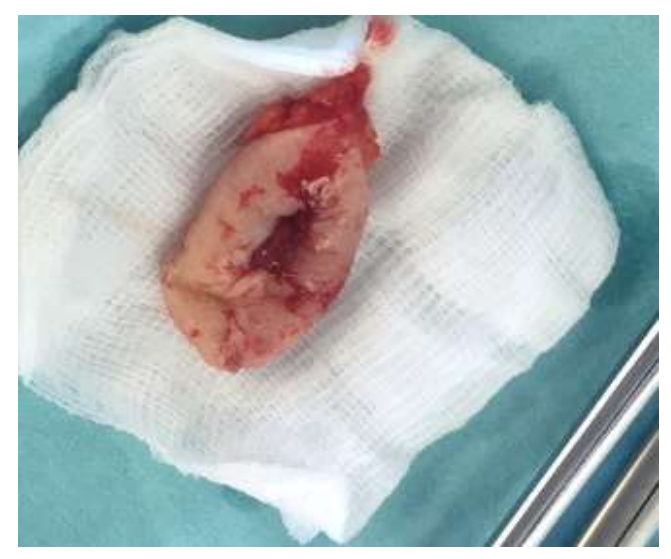

Fig.2. Fistula dissected

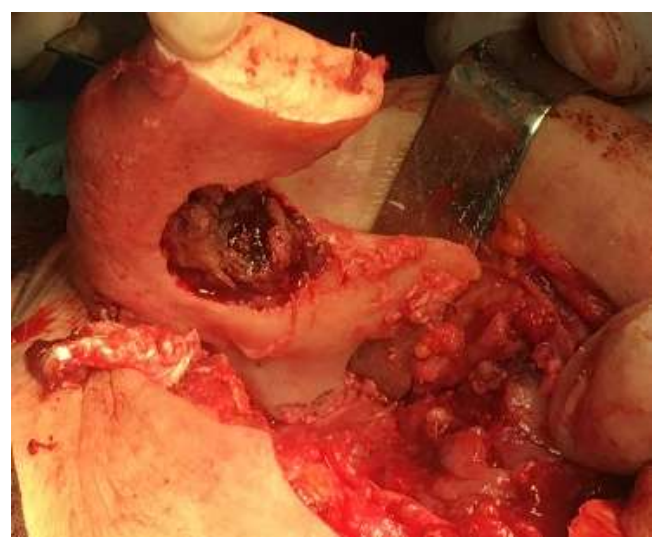

Fig.3. Hemiexarticulation 


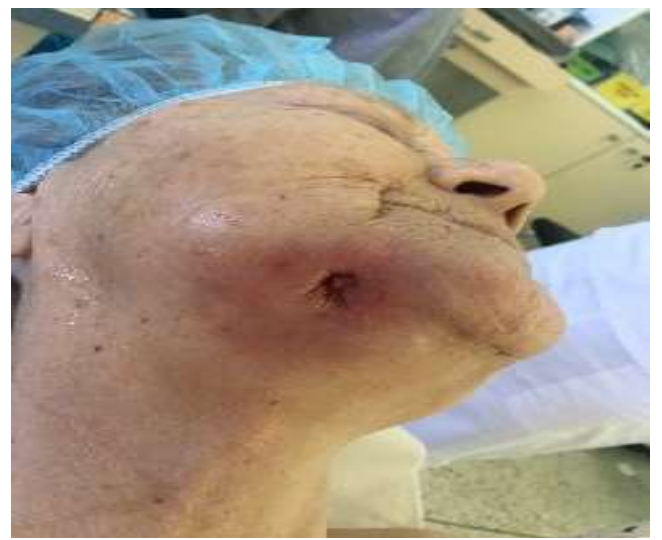

Fig.4. Resected lower jaw

\section{DISCUSSION}

MRONJ treatment is generally difficult and the optimal treatment strategy has not yet been established.In particular, it is not known whether the affected persons should receive symptomatic therapy with pain and infection control and possibly minimally invasive debris of the surface of the necrotic bone, or resective surgical methods should be used[3]. The subject is complicated because some patients have minimal symptoms and can therefore benefit from minimally invasive therapy. In contrast, there are others, with a painful, advanced disease that does not respond to symptomatic therapy and require more aggressive intervention [3]. For this reason, prevention is even more important. A multidisciplinary team approach is proposed, including a dentist, oncologist and maxillofacial surgeon to evaluate and decide on the best therapy for the patient [7]. The choice between conservative treatment and surgery is not easy and must be made on a case-by-case basis. In our case, the team desided on surgery involving hemiexarticulation.

Medicinal osteonecrosis of jaw-related drugs is used to treat conditions associated with cancer, as well as skeletal malignancy related to skeletal, associated with bone tumor metastases, and to manage lytic lesion associated with multiple myeloma. [8]. Patients with osteoporosis using anti-resorptive drugs (ARD) may have some side effects such as esophagitis, musculoskeletal pain, hypocalcemia, ocular inflammation, and ONJ-related bisphosphonates (BRONJ), however the drug is well tolerated.

Anti-resorptives include five major classes of agents: bisphosphonates, estrogens, selective estrogen receptor modulators, calcitonin, and monoclonal antibodies such as Denosumab. ARDs are the most widely used first line antiresorptive therapy and are recommended for patients with osteoporosis and individuals at high risk for fracture [8].

The pathophysiology of MRONJ has not been fully understood and effective and appropriate therapy remains to be decided. In the future, it is essential to improve the current knowledge of MRONJ and to develop better strategies for its prevention and treatment [1].

\section{CONClusion}

Patients taking osteoporosis or cancer medicines should receive dental care because of the risk of invasive jawbone procedures for patients taking these drugs, which may cause osteonecrosis of the jaw. When diagnosed, osteonecrosis of the jaw should be surgically treated in connection with antibiotic therapy and patients should be monitored.

Osteonecrosis of the jaw is associated with antiresorption and anti-angiogenic drugs, this correlation is accepted unanimously and due to those patients using these drugs more attention should be paid to oral care.

This patient report underwent surgery to remove the affected tissue. The patient was followed up for 8 months of surgery without any signal of complications.

Management of MRONJ may have a similar purpose to that of basic cancer treatment, namely to reduce symptoms and infection, and to improve quality of life rather than "cure" necrosis. All possible sources of infection in the oral cavity should be sought in any patient who is contemplated to receive antiresorptive therapy. 


\section{CONFLICT OF INTERESTS}

The authors have no conflict of interest to declare.

\section{REFERENCES}

[1] Hristamyan MA. Risk Factors of Bisphosphonate-Associated Osteonecrosis of the Jaws: Review of the Literature. ARC Journal of Dental science. 2018; 3(1): 6-10

[2] Stockmann P, Hinkmann FM, Lell MM, Fenner M, Vairaktaris E, Neukam FW, Nkenke E. Panoramic radiograph, computed tomography or magnetic resonance imaging. Which imaging technique should be preferred in bisphosphonateassociated osteonecrosis of the jaw? A prospective clinical study. Clin Oral Investig. 2010 Jun;14(3):311-7.

[3] Hristamyan M, Stoilova Y, Pechalova P, Hristamyan V. Analysis of the knowledge of the epidemiology and clinic of bisphosphonaterelated osteonecrosis of the jaws , Bulgarian medicine. 2017 7(2);46-56

[4] Viviano M, Rossi M, Cocca S. A rare case of osteonecrosis of the jaw related to imatinib. $\mathbf{J}$
Korean Assoc Oral Maxillofac Surg. 2017 Apr;43(2):120-124.

[5] Egloff-Juras C, Gallois A, Salleron J, Massard V, Dolivet G, Guillet J, Phulpin B. Denosumabrelated osteonecrosis of the jaw: A retrospective study. J Oral Pathol Med. 2018 Jan;47(1):66-70.

[6] Aljohani S, Fliefel R, Ihbe J, Kühnisch J, Ehrenfeld M, Otto S. What is the effect of antiresorptive drugs (ARDs) on the development of medication-related osteonecrosis of the jaw (MRONJ) in osteoporosis patients: A systematic review. J Craniomaxillofac Surg. 2017 Sep;45(9):1493-1502.

[7] Chen JS, Sambrook PN. Antiresorptive therapies for osteoporosis: a clinical overview. Nat Rev Endocrinol. 2011 Sep 6;8(2):81-91.

[8] Reginster JY, Neuprez A, Beaudart C, Lecart MP, Sarlet N, Bernard D, Disteche S, Bruyere O. Antiresorptive drugs beyond bisphosphonates and selective oestrogen receptor modulators for the management of postmenopausal osteoporosis. Drugs Aging. 2014 Jun;31(6):413-24.

Citation: Tsolov RB, Yordanov GY, Dushkova TS. The Case of Lower Jaw Resection due to MedicationInduced Osteonecrosis Mronj. ARC Journal of Dental science. 2020; 5(1):11-14. doi:dx.doi.org/10.20431/2456-0030.0501004.

Copyright: () 2020 Authors. This is an open-access article distributed under the terms of the Creative Commons Attribution License, which permits unrestricted use, distribution, and reproduction in any medium, provided the original author and source are credited. 